\title{
Football team performance efficiency and effectiveness in a corruptive context: the Calciopoli case
}

\author{
Giambattista Rossi, Dries Goossens, Gian Luca Di Tanna, Francesco Addesa
}

European Sport Management Quarterly

DOI: $10.1080 / 16184742.2018 .1553056$

Research question: While there has been considerable research on production and efficiency in most of the major European football leagues, corresponding evidence relating to the Italian Serie $\mathrm{A}$ is limited. To address this imbalance, this paper analyses the technical efficiency and effectiveness of Italian football clubs, taking into consideration the impact of the Calciopoli corruption scandal in 2006. This allows assessing how clubs' efficiency and effectiveness were affected by the points deduction inflicted on the clubs involved in the scandal.

Research methods: Utilising a panel dataset comprising season aggregated match statistics over ten seasons from 2000/01 to 2009/10, this paper estimates a production function for the league and, then, calculates the relative offensive and defensive efficiency and the football effectiveness of 36 teams. To achieve this, Data Envelopment Analysis (DEA) models have been used to calculate the frontiers of efficient production.

Results and findings: The results partially confirm the evidence of the literature but they also reveal how playing style changed, emphasizing the importance of attacking play at the expense of defensive play in Italian Serie A. Clubs that suffered a points deduction following their involvement in the Calciopoli scandal changed their usual tactical behaviour, presumably to compensate for the impact of these punishments.

Implications: League authorities should be aware that the clubs' performance has been affected following the Calciopoli scandal sanctions, which may have contributed to the spectator decline. Furthermore, we identify offensive efficiency as the key determinant of team success, which is a helpful insight for team management with respect to their transfer and salary policy.

Keywords: Efficiency; DEA; Corruption; Italian football 


\section{Introduction}

In Italy, professional football has been historically the largest leisure activity, with vast social and economic importance. During the 2012/13 season, professional football registered a cumulative turnover equal to almost $€ 2.7 \mathrm{bn}$ with a direct impact on the Italian economy equal to 0.15 per cent of GDP, and a total contribution of $€ 1.03 \mathrm{bn}$ in tax revenues (Arel, PricewaterhouseCoopers, \& Federcalcio, 2012). Conversely, professional football has declined constantly and slowly commercially since the early 2000s, due to clubs' rising payrolls, accounting for 90 per cent of total costs since the 2004/05 season and spent to attract worldwide football talent, and to a slow revenues' growth compared to the other major European leagues (Baroncelli \& Lago, 2006; Boeri \& Severgini, 2012; Bof, Montanari, \& Silvestri, 2008). Accounting data revealed an increasing trend in net losses equal to roughly $€ 250 \mathrm{~m}$ per year, with a seven per cent annual growth rate in operational losses. The total debt of Serie A clubs has increased with nine per cent per year; i.e. by over 60 per cent since 2006/07. On the revenue side, while in 2011 media revenues steadily represented around 56 per cent of total revenues, gate revenues were decreasing. Consequently, Serie A clubs have been predictably subject to a high mortality rate: nine out of 37 Serie A teams went bankrupt from 2001 to 2011 (Boeri \& Severgini, 2012).

This paper considers the production function of Serie A Italian football clubs, looking at their operating efficiency and football effectiveness to analyse their performance during the last decade. Since the seminal studies of Rottenberg (1956) and Scully (1974) on baseball, a vast literature analysing production functions of sports clubs has been carried out by several scholars. Identifying a production function can help chairmen, managers and coaches to manage several issues, it estimates the key determinants of team success and how individual players contribute to that success, helping teams with match selection and preparation, besides tactical decisions and changes. It can also guide negotiations on player salaries, along with recognizing those areas in which a team can improve its future performance, including its playing style and transfer market strategy. While such considerations can affect an individual club's commercial and financial success, and enhance revenue sources, analysing the production function is also relevant for a sport's organizational structure and managerial decision making, as it assists the ruling body making an attractive and successful product, seen in terms of public interest, media coverage and revenues, and profitable sponsorship agreements. 
This paper contributes to the research strand that clearly concentrates on the straight relationships between on-field team success and the aggregate contribution of players' skills and abilities in terms of their football performance. First, whilst production function investigation for Serie A has been limited, this analysis is based on a panel dataset comprising season statistics for 36 Serie A clubs over ten seasons. Second, the time period and the related data include the seasons when the league was discredited by the Calciopoli scandal, which allow us to model the effects of the subsequent sanctions on the involved clubs' behaviour. Finally, this work estimates the production functions using non-parametric techniques with mathematical models, specifically Data Envelopment Analysis (DEA) models that calculate the frontier efficient production for given productive factors. The empirical results obtained differentiate between offensive and defensive production along a period of 10 seasons and explicitly include the Calciopoli scandal. In this way, we can attempt to answer with greater precision how Italian football has changed and what kind of impact corruption punishments have had on it.

Aside from this introductory part, this paper is structured as follows. First, a brief review of the existing literature on sporting production functions is provided. Then, the league structure of Italian professional football and the key features of the Calciopoli scandal are explained, followed by the adopted theoretical framework. While the fifth part describes the dataset and the model specification used for this paper, the sixth part presents the empirical results obtained with DEA analysis - looking at offensive and defensive efficiencies and football effectiveness in Serie A over ten seasons and how these were affected by the Calciopoli scandal. Finally, based on the efficiency and effectiveness analyses as a reference, we provide a plausible explanation of the final rankings, followed by managerial implications and conclusions.

\section{Literature review}

From a management perspective, the production function of any organisation is seen as the technical relationship between productive inputs and their relative contribution to output. Rottenberg (1956) was the first scholar to conceive that a sports team, like any other enterprise, offers a product, victory or success, by combining and using different inputs, seen as the skills and other characteristics of the team. Accordingly, Scully (1974) conducted the first empirical study that formally estimated and employed a production function to compare wages and Major League Baseball players' marginal revenue product 
in order to assess the level of monopsonistic exploitation. Since then, this method is recognised as the standard methodology in sporting production function studies (Scully, $1989 ; 1995)$.

The early studies involved US-based sports, whose data-richness allows categorizing individual contributions and measurable match play statistics (Atkinson, Stanley, \& Tschirart, 1988; Chatterjee, Campbell, \& Wiseman, 1994; Krautmann, 1990; McCormick \& Clement, 1992; Porter \& Scully, 1982; Ruggiero, Hadley, \& Gustafson, 1996; Scott, Long, \& Somppi, 1985; Zak, Huang, \& Sigfried, 1979; Zech, 1981). The relative scarcity of empirical research on other professional sports in different nations is explained by their intrinsic nature, like rugby and football, whose interaction between teams and complementarity of player contributions within teams is significant (Carmichael \& Thomas, 1995; Schofield, 1988). The increased availability and sophistication of quantifiable data, such as the detailed player performance statistics, provide invaluable datasets for analysis. This opportunity has recently favoured the growth of production function studies across sports and continents and the related research strand treating efficiency aspects with various specific applications, particularly featuring the assessment of coaching/managerial efficiency (Dawson \& Dobson, 2002; Dawson, Dobson, \& Gerrard, 2000; Frick \& Simmons, 2008).

Production functions are also distinguished according to the selection of output and input measures, and the estimation method. In football, while output is usually measured by points won, league position, win rates, and goals or goals difference, input measures include attacking and constructive play, aggressive and defensive play and non-playing aspects, including managerial inputs (Barros \& Leach, 2006a; Barros \& Leach, 2006b; Dawson, Dobson, \& Gerrard, 2000; Espitia-Escuer \& García-Cebríán, 2004; Gerrard, 2006). Concerning the estimation method, there are two distinct approaches (Collier, Johnson, \& Ruggiero, 2011): the econometric stochastic frontier approach (SFA) based on tools and concepts from regression analysis and the deterministic non-parametric frontier methodology, including Data Envelopment Analysis (DEA), built on axiomatic properties and mathematical programming techniques.

To summarise, while several studies that specifically treat efficiency measurement predominantly examine English and Spanish football (Barros, Del Corral, \& García-delBarrio, 2008; Barros \& García-del-Barrio, 2008; Barros, García-del-Barrio, \& Leach, 2009; Barros \& Leach, 2006a; Barros \& Leach, 2006b; Barros \& Leach, 2007; Carmichael, Thomas, \& Ward, 2001; Espitia-Escuer \& García-Cebrían 2004; Espitia-Escuer \& García-Cebríán, 
2006; Espitia-Escuer \& García-Cebrián, 2008; García-Sanchez, 2007; Gómez \& Picazo-Tadeo, 2010; González- Sala-Garrido, Carrión, Esteve, \& Boscá, 2009; Guzmán \& Morrow, 2007; Haas, 2003a) with a few using data from Brazil (Barros, Assaf, \& Sá-Earp, 2010), Germany (Haas, Kocher, \& Sutter, 2004; Tiedemann, Francksen, \& Latacz-Lohmann, 2011) and the US (Haas, 2003b), Italian football has been analysed over three seasons using DEA only by Boscá, Liern, Martinez, and Sala (2009). They conclude that defensive efficiency is much more important than offensive efficiency to obtain a good final ranking in Serie A. Our study incorporates a richer set of direct performance measures that reduces the need to use proxy measures to represent particular aspects, such as defensive performance. Moreover, it focuses on a relatively long time period with more club-observations and covers the seasons when the Calciopoli scandal erupted.

\section{The Calciopoli Scandal}

Since its establishment in 1898, Serie A represents the top division of Italian football under the supervision of the FIGC, the Italian football association, which manages the operation at professional and amateur levels. Nowadays, Serie A is separately run by Lega Serie A, composed of 20 clubs that compete for the championship title, the so-called "Scudetto". The Serie A league winner, the clubs finishing $2^{\text {nd }}$ and $3^{\text {rd }}$ in the final Serie A table directly qualify for next season's UEFA Champions League. Clubs ranked $4^{\text {th }}$ to $6^{\text {th }}$ compete in the following season's UEFA Europa League, together with the winner of the domestic knock-out cup competition, the so-called "Coppa Italia". In 2003 Serie A faced a league restructure (Hamil, Morrow, Idle, Rossi, \& Faccendini, 2010), when Catania Calcio, a Serie B club, was involved in player eligibility controversy. The dispute led to the expansion of both Serie A from 18 to 20 teams and Serie B from 20 to 22 teams. During the study period from 2000/01 to 2009/10, 33 different clubs in total competed in Serie A and 12 teams achieved a top six position at least once. Four different teams, featuring in all ten seasons, won the Scudetto, and three other clubs appeared in the top six on all but one occasion.

Because of poor practice in corporate governance and administration, Italian football has faced numerous major scandals, linked to doping, fake passports, bribery and match-fixing (Agnew, 2007; Di Meo and Ferraris, 2012; Foot, 2007; Jones, 2007). Arguably the most detrimental and relevant scandal is known as Calciopoli in 2006, which erupted shortly before the FIFA World Cup in Germany. Supported by scrupulous investigations, the Italian police discovered a network of close relationships that the involved certain clubs', 
leagues' and associations' officials influencing the organizational selection and appointment of "amicable" referees for specific matches with the intention of fixing their results (Boeri, T. and Severgini, 2011; Hamil et al., 2010). Five Serie A clubs - FC Juventus, AC Milan, ACF Fiorentina, SS Lazio and Reggina Calcio - and one Serie B, AC Arezzo, were involved and received club-level punishments while several officials at different levels were also banned from Italian football for specific periods (Hamil et al., 2010). FC Juventus was demoted to Serie B with a nine-point deduction for the following season and retrospectively stripped of its 2004/05 and 2005/06 Serie A titles. AC Milan, ACF Fiorentina, SS Lazio and Reggina Calcio suffered a deduction of 8, 15, 13 and 11 points respectively in the 2006/07 Serie A Season. Although this scandal was not the only manifestation of corruption in Italian football, it is the only one in recent history that resulted in point deductions in the subsequent season for the involved teams, instead of only administrative sanctions after the season (Hamil et al., 2010).

Besides the short-lived sporting effects of the clubs' punishments, the Calciopoli scandal widely affected Italian football, with particular regard to attendance figures. During the study period, the Serie A average attendance per match was below 25,000 spectators; the lowest among the top European leagues. Beside high ticket prices and excessive TV exposure of football, Italian football was also negatively affected by numerous episodes of violence and hooliganism occurring in Italian stadia and in their proximity (Caruso \& Di Domizio, 2012). Corruption issues highlighted by the Calciopoli scandal accelerated the decline in gate revenues and deteriorated the balance sheets for all the clubs directly involved (Babatunde, Migali, \& Simmons, 2012). Consequently, other teams faced a negative spillover on attendance that was partially compensated by rising income from sales of television broadcast rights.

\section{Theoretical framework}

From a theoretical perspective, this paper follows the research strand that studies the production function in terms of the overall performance as a result of an input-output relationship. In general terms, the existence and the development of any organisation depends on the achievement of adequate results in their operation and behaviour (Brittan, 1997; Korten, 1998; Magretta, 2000). In order to achieve such results, an organisation needs to fulfil two basic conditions; i.e. to adequately use the available (given and potential) resources and to meet the needs and requirements of the customers (Porter, 1985; Modis, 1998; Scott, 2000). In other 
words, any organisation depends on the achievement of the requisite operating efficiency and operating effectiveness of its operations and behaviour as the two components of the overall performance. In the management literature (Chan, 2003; Druker; 1963), operating efficiency refers to "doing things right" as the organization's ability to achieve the output considering the minimum inputs level. Additionally, operating effectiveness means "doing the right things" and choices of activities in a proper way as it measures the firms' ability to gain prearranged objectives and goals (Druker, 1963; Ken et al., 2006).

In the football context, we build on the notion of "sports production function", which assumes that teams, like other enterprises, adopt a production process, with "output" measured as sporting success, combining different playing and non-playing inputs. Carmichael et al. (2010) find a strong relation between sporting success and commercial success, as well as evidence that clubs reinvest the revenue they earned from seasonal success into investments in playing skills, which are in turn rewarded by further sporting success. Hence, football effectiveness can be seen as the points accumulated at the end of the season, as this determines a team's final league ranking. Football effectiveness obviously depends on the individual match results, which is in turn decided by the goals scored for and against. This means that, on a match level, any football team has to maximise the number of goals scored and to minimize the number of goals conceded given their available skills. Therefore, we talk about operating offensive efficiency - which relates football players' offensive performance to the number of goals scored -, and operating defensive efficiency - whose relationship links football players' defensive performance to (the inverse of) the number of goals conceded.

Figure 1: Model for operating efficiency and operating effectiveness in football

\begin{tabular}{lll} 
Inputs & Throughput & Outputs \\
$\begin{array}{l}\text { PLAYERS' OFFENSIVE } \\
\text { PERFORMANCE }\end{array}$ & SCORED & \\
\hline GOALS & \\
OPERATING OFFENSIVE EFFICIENCY & ACCUMULATED \\
PLAYERS' DEFENSIVE & 1/RECEIVED \\
PERFORMANCE & GOALS & \\
OPERATING DEFENSIVE EFFICIENCY &
\end{tabular}


As shown in Figure 1, this approach enables us to identify the strong and weak points of each team with respect to the tactical skills of its offence and defence, its effectiveness or precision of play which is strongly connected to the creation of present and future revenues (GarcíaSanchez; 2007). Ho and Zho (2004) introduced a model with two stages to obtain overall performance. Efficiency and effectiveness scores are then obtained with a DEA model separately. Thus, in the first stage, the players' talent is used to produce two throughputs, one that is positive (goals scored) and another that is negative (goals conceded) to be minimised, which independently determine the efficiency of the offence and defence of a football team. Both efficiencies are introduced into the second stage to produce the final output, the points obtained by a team at the end of the season, evaluating the importance of each style of play by the offence or defence - in the operating effectiveness.

Within this theoretical framework, we consider the Calciopoli scandal as a structural break as it altered sensibly the economic and competitive conditions of the Italian Serie A for the coming years (Boeri and Severgnini, 2012). As the scandal involved point-deduction sanctions to involved Serie A clubs, we assume that the top Italian league has been affected. In this unique and peculiar case, we test whether the punishments of point-deductions have pushed Serie A clubs to decline their performance in terms of operating efficiency and football effectiveness and, additionally, we identify how they changed their playing style. If this hypothesis is ascertained, we can explain that the spectator decline during the 2005/06 season was partially due to deterioration of clubs' performance.

\section{Methodological aspects and data}

As mentioned previously, two classes of methods to estimate efficiencies prevail. SFA uses observed input-output correspondences to estimate an underlying relationship between the inputs and outputs. This function is then used as the frontier against which to measure the efficiencies. On the other hand, DEA is a non-parametric approach based on linear programming which takes the observed input and output data to estimate the frontier (rather than explicitly stating the form of the frontier). In the context of football, we assume that, although the levels of operating efficiency and football effectiveness differ between clubs (as their management and organisation structure varies), the levels of technology in terms of tactics, training and physical preparation are similar and homogenous for all clubs. Given this assumption, as argued by Boscá et al. (2009), a non-parametric methodology, specifically DEA, is the most suitable optimisation technique, as it provides great flexibility and an 
absence of specification errors because no particular functional form is needed. Conversely, the disadvantage of being technically deterministic results in a bias of the efficiency results and the attribution of any random shock to inefficiency due to the presence of atypical observations.

As clearly summarized by Villa and Lozano (2016), DEA methods have been applied very successfully in the football context using CCR and BBC type of models. In function of the output chosen and following the mainstream approach for this specific output, we have decided to adopt the CCR-O model $^{1}$ to assess the potential of each team in the Italian professional football league between 2000 and 2010. Despite the availability of panel data, the data correspond to each season separately as our main purpose is to evaluate teams' efficiency and effectiveness and relate them to the context of the Calciopoli scandal in 2006. Moreover, our analysis distinguishes between offensive and defensive production to calculate separately offensive and defensive efficiency indicators, as the measurement of output combines offensive productivity (goal-scoring) with defensive efficiency (preventing goals). As a result, we combine inputs as of indicators of each club's offensive and defensive efficiency in line with their expected signs in the regressions with club's points won and, then, we calculate the frontier of effective production. The analysis of offensive and defensive efficiency looks at the input-output of the teams with highest outputs per each input and compares the productivities of the remaining teams with these.

Our dataset was supplied by Digital Soccer - the official data supplier of Lega Serie A - and a wide variety of match performance data were included and used in our analysis of the offensive and defensive efficiency and effectiveness of Italian Serie A clubs from season 2000/01 to 2009/10. Table 1 summarises the dataset for a varied mix of performance indicators aggregated at club level. As the number of teams participating has changed from 18 to 20 since season 2004/05, the table shows the average and the standard deviation for the different output and input measures standardised for the number of games played. Nevertheless, despite the ten season data period, the maximum number of observations recorded in any one season is limited to 20 teams.

\footnotetext{
${ }^{1}$ Implemented using GAMS and solved with CPLEX.
} 
Table 1: Offensive and defensive inputs in the Italian Serie A (2000-2010)

\begin{tabular}{|c|c|c|c|c|}
\hline Variables & Mean & Std. Dev. & Min. & Max. \\
\hline \multicolumn{5}{|l|}{ Offensive inputs per game } \\
\hline Goals & 1.303 & 0.341 & 0.617 & 2.236 \\
\hline Shots & 13.073 & 2.076 & 9.21 & 19.868 \\
\hline Shots on target & 4.94 & 0.968 & 2.911 & 10.342 \\
\hline Assists & 0.83 & 0.256 & 0.294 & 1.473 \\
\hline Counterattacks & 0.014 & 0.002 & 0.005 & 0.02 \\
\hline Crosses completed & 3.739 & 0.769 & 1.921 & 5.578 \\
\hline Crosses ratio & 0.006 & 0.0007 & 0.003 & 0.008 \\
\hline Crosses total & 16.79 & 2.813 & 10.65 & 24.41 \\
\hline Passes completed & 311.9 & 54.23 & 96.02 & 476.18 \\
\hline Total balls touched & 536.65 & 55.68 & 399.32 & 710.71 \\
\hline Useful dribbles & 8.348 & 2.084 & 3.973 & 14.91 \\
\hline \multicolumn{5}{|l|}{ Defensive inputs per game } \\
\hline Goals conceded & 1.303 & 0.293 & 0.558 & 2.058 \\
\hline Opponents off-sides & 3.148 & 1.044 & 1.289 & 6.421 \\
\hline Clearances & 4.062 & 1.007 & 2.029 & 8.558 \\
\hline Interceptions & 100.94 & 7.317 & 81.65 & 121.39 \\
\hline Anticipations & 17.25 & 3.504 & 9.947 & 31.08 \\
\hline Recovered balls & 160.18 & 12.27 & 132.21 & 188.79 \\
\hline Ratio goals conceded to saves & 0.012 & 0.002 & 0.006 & 0.028 \\
\hline Saves & 3.286 & 0.535 & 1.617 & 4.705 \\
\hline Goalkeepers catches & 7.204 & 0.959 & 4.5 & 9.382 \\
\hline Tackles & 19.806 & 2.85 & 12.97 & 29.08 \\
\hline Yellow cards & 2.154 & 0.433 & 0.263 & 3.105 \\
\hline Red cards & 0.157 & 0.0723 & 0.026 & 0.5 \\
\hline Fouls committed & 19.617 & 2.563 & 13.44 & 27 \\
\hline
\end{tabular}

Table 2: Offensive performance correlation table (shotstar = shots on target; coattacks = counterattacks; crosscomp $=$ crosses completed, i.e. received by own team player; crossrat= ratio of crosses completed to crosses made; pscomp = passes completed, i.e. received by own team player; totch $=$ balls touched; drus $=$ useful dribbles)

\begin{tabular}{|c|c|c|c|c|c|c|c|c|c|c|}
\hline & Goals & Shots & Shotstar & Assists & Conattacks & Crosscomp & Crossrat & Crosses & Pscomp & Totch \\
\hline Shots & $\begin{array}{c}0.6463 \\
(0.0000)\end{array}$ & & & & & & & & & \\
\hline Shotstar & $\begin{array}{c}0.7237 \\
(0.0000)\end{array}$ & $\begin{array}{c}0.8484 \\
(0.0000)\end{array}$ & & & & & & & & \\
\hline Assists & $\begin{array}{c}0.9072 \\
(0.0000)\end{array}$ & $\begin{array}{c}0.5947 \\
(0.0000)\end{array}$ & $\begin{array}{c}0.7083 \\
(0.0000)\end{array}$ & & & & & & & \\
\hline Coattacks & $\begin{array}{c}0.5585 \\
(0.0000)\end{array}$ & $\begin{array}{c}0.0287 \\
(0.6926)\end{array}$ & $\begin{array}{c}0.3505 \\
(0.0000)\end{array}$ & $\begin{array}{c}0.5929 \\
(0.0000)\end{array}$ & & & & & & \\
\hline Crosscomp & $\begin{array}{c}0.3187 \\
(0.0000)\end{array}$ & $\begin{array}{c}0.4249 \\
(0.0000)\end{array}$ & $\begin{array}{c}0.4434 \\
(0.0000)\end{array}$ & $\begin{array}{c}0.3479 \\
(0.0000)\end{array}$ & $\begin{array}{c}0.2193 \\
(0.0022) \\
\end{array}$ & & & & & \\
\hline Crossrat & $\begin{array}{c}0.1667 \\
(0.0208)\end{array}$ & $\begin{array}{c}0.0928 \\
(0.2007)\end{array}$ & $\begin{array}{c}0.1506 \\
(0.0371)\end{array}$ & $\begin{array}{c}0.1958 \\
(0.0065)\end{array}$ & $\begin{array}{c}0.3425 \\
(0.0000)\end{array}$ & $\begin{array}{c}0.6289 \\
(0.0000)\end{array}$ & & & & \\
\hline Crosses & $\begin{array}{c}0.2818 \\
(0.0001)\end{array}$ & $\begin{array}{c}0.4538 \\
(0.0000)\end{array}$ & $\begin{array}{c}0.4335 \\
(0.0000)\end{array}$ & $\begin{array}{c}0.2799 \\
(0.0001)\end{array}$ & $\begin{array}{c}0.1604 \\
(0.0262)\end{array}$ & $\begin{array}{c}0.8460 \\
(0.0000)\end{array}$ & $\begin{array}{c}0.1845 \\
(0.0104)\end{array}$ & & & \\
\hline Pscomp & $\begin{array}{c}0.6470 \\
(0.0000)\end{array}$ & $\begin{array}{c}0.6242 \\
(0.0000)\end{array}$ & $\begin{array}{c}0.3674 \\
(0.0000)\end{array}$ & $\begin{array}{c}0.6471 \\
(0.0000)\end{array}$ & $\begin{array}{c}0.3449 \\
(0.0000)\end{array}$ & $\begin{array}{c}0.4865 \\
(0.0000)\end{array}$ & $\begin{array}{c}0.2406 \\
(0.0008)\end{array}$ & $\begin{array}{c}0.4837 \\
(0.0000)\end{array}$ & & \\
\hline Totch & $\begin{array}{c}0.6321 \\
(0.0000)\end{array}$ & $\begin{array}{c}0.6687 \\
(0.0000)\end{array}$ & $\begin{array}{c}0.6915 \\
(0.0000)\end{array}$ & $\begin{array}{c}0.6315 \\
(0.0000)\end{array}$ & $\begin{array}{c}0.3385 \\
(0.0000)\end{array}$ & $\begin{array}{c}0.4951 \\
(0.0000)\end{array}$ & $\begin{array}{c}0.2448 \\
(0.0006)\end{array}$ & $\begin{array}{c}0.5041 \\
(0.0000)\end{array}$ & $\begin{array}{c}0.8826 \\
(0.0000)\end{array}$ & \\
\hline Drus & $\begin{array}{c}0.3488 \\
(0.0000)\end{array}$ & $\begin{array}{c}0.4176 \\
(0.0000)\end{array}$ & $\begin{array}{c}0.4000 \\
(0.0000)\end{array}$ & $\begin{array}{c}0.2773 \\
(0.0001)\end{array}$ & $\begin{array}{c}0.2867 \\
(0.0001)\end{array}$ & $\begin{array}{c}0.2209 \\
(0.0021)\end{array}$ & $\begin{array}{c}0.2179 \\
(0.0024)\end{array}$ & $\begin{array}{c}0.2613 \\
(0.0003)\end{array}$ & $\begin{array}{c}0.4608 \\
(0.0000)\end{array}$ & $\begin{array}{c}0.5525 \\
(0.0000)\end{array}$ \\
\hline
\end{tabular}


Table 3: Defensive performance correlation table $($ Invgoalscon = Inverse of goals conceded; Oppshots = Shots by the opponent; Clear = clearances; Oppofsde = Opponents offsides; Inter = Interceptions; Ant = Anticipations; Recbal $=$ Recovered balls; Effrecbal = Effective recovered balls; Temrecbal = Team effective recovered balls; Gcgksv $=$ Ratio goals conceded to saves; Gkct $=$ Goalkeepers catches; Yellow $=$ Yellow cards; Red $=$ Red cards; Fouls $=$ Fouls committed $)$

\begin{tabular}{|c|c|c|c|c|c|c|c|c|c|c|c|c|c|c|c|c|c|}
\hline & $\begin{array}{c}\text { Oppshot } \\
\text { s }\end{array}$ & Clear & $\begin{array}{c}\text { Oppshot } \\
\text { s }\end{array}$ & Clear & $\begin{array}{c}\text { Oppofsd } \\
\mathrm{e}\end{array}$ & Inter & Ant & Recbal & $\begin{array}{c}\text { Effrecba } \\
1\end{array}$ & $\begin{array}{c}\text { Temrecb } \\
\text { al }\end{array}$ & Runs & Gcgksv & Saves & Gkct & Tackles & Yellow & Red \\
\hline $\begin{array}{c}\text { Invgoals } \\
\text { con }\end{array}$ & $\begin{array}{l}-0.4837 \\
(0.0000)\end{array}$ & & & & & & & & & & & & & & & & \\
\hline $\begin{array}{c}\text { Oppshot } \\
\text { s }\end{array}$ & $\begin{array}{c}-0.1272 \\
(0.0787) \\
\end{array}$ & $\begin{array}{c}0.2089 \\
(0.0036) \\
\end{array}$ & & & & & & & & & & & & & & & \\
\hline Clear & $\begin{array}{l}-0.0025 \\
(0.9724) \\
\end{array}$ & $\begin{array}{l}-0.3900 \\
(0.0000) \\
\end{array}$ & $\begin{array}{c}0.1157 \\
(0.1101) \\
\end{array}$ & & & & & & & & & & & & & & \\
\hline $\begin{array}{c}\text { Oppofsd } \\
\mathrm{e}\end{array}$ & $\begin{array}{c}0.0096 \\
(0.8947) \\
\end{array}$ & $\begin{array}{l}-0.0205 \\
(0.7774) \\
\end{array}$ & $\begin{array}{c}0.0942 \\
(0.1936) \\
\end{array}$ & $\begin{array}{c}0.0705 \\
(0.3313) \\
\end{array}$ & & & & & & & & & & & & & \\
\hline Inter & $\begin{array}{l}-0.1476 \\
(0.0411) \\
\end{array}$ & $\begin{array}{c}0.1835 \\
(0.0109) \\
\end{array}$ & $\begin{array}{c}0.5589 \\
(0.0000) \\
\end{array}$ & $\begin{array}{c}0.0341 \\
(0.6391) \\
\end{array}$ & $\begin{array}{c}0.1213 \\
(0.0938) \\
\end{array}$ & & & & & & & & & & & & \\
\hline Ant & $\begin{array}{l}-0.0610 \\
(0.4008) \\
\end{array}$ & $\begin{array}{c}0.1143 \\
(0.1145) \\
\end{array}$ & $\begin{array}{c}0.4563 \\
(0.0000)\end{array}$ & $\begin{array}{c}0.1210 \\
(0.0945) \\
\end{array}$ & $\begin{array}{c}0.3170 \\
(0.0000)\end{array}$ & $\begin{array}{c}0.6772 \\
(0.0000) \\
\end{array}$ & & & & & & & & & & & \\
\hline Recbal & $\begin{array}{r}0.0860 \\
(0.2592) \\
\end{array}$ & $\begin{array}{r}-0.0823 \\
(0.2801) \\
\end{array}$ & $\begin{array}{c}0.2329 \\
(0.0020) \\
\end{array}$ & $\begin{array}{c}0.0594 \\
(0.4360) \\
\end{array}$ & $\begin{array}{c}0.7290 \\
(0.0000) \\
\end{array}$ & $\begin{array}{c}0.5202 \\
(0.0000) \\
\end{array}$ & $\begin{array}{c}0.9356 \\
(0.0000) \\
\end{array}$ & & & & & & & & & & \\
\hline $\begin{array}{c}\text { Effrecba } \\
1\end{array}$ & $\begin{array}{l}-0.2437 \\
(0.0012) \\
\end{array}$ & $\begin{array}{c}0.2750 \\
(0.0002) \\
\end{array}$ & $\begin{array}{c}0.2201 \\
(0.0035)\end{array}$ & $\begin{array}{l}-0.0101 \\
(0.8949) \\
\end{array}$ & $\begin{array}{c}0.5404 \\
(0.0000) \\
\end{array}$ & $\begin{array}{c}0.4699 \\
(0.0000)\end{array}$ & $\begin{array}{c}0.7750 \\
(0.0000) \\
\end{array}$ & $\begin{array}{c}0.5020 \\
(0.0000)\end{array}$ & & & & & & & & & \\
\hline $\begin{array}{c}\text { Temrecb } \\
\text { al }\end{array}$ & $\begin{array}{c}0.1847 \\
(0.0139) \\
\end{array}$ & $\begin{array}{c}-0.1088 \\
(0.1493) \\
\end{array}$ & $\begin{array}{c}0.3800 \\
(0.0000)\end{array}$ & $\begin{array}{c}0.1291 \\
(0.0867) \\
\end{array}$ & $\begin{array}{c}0.3800 \\
(0.0000) \\
\end{array}$ & $\begin{array}{c}0.5790 \\
(0.0000) \\
\end{array}$ & $\begin{array}{c}0.7119 \\
(0.0000) \\
\end{array}$ & $\begin{array}{c}0.7436 \\
(0.0000) \\
\end{array}$ & $\begin{array}{c}0.2184 \\
(0.0057) \\
\end{array}$ & & & & & & & & \\
\hline Runs & $\begin{array}{l}-0.1697 \\
(0.0186) \\
\end{array}$ & $\begin{array}{c}0.5626 \\
(0.0000) \\
\end{array}$ & $\begin{array}{c}0.2246 \\
(0.0017) \\
\end{array}$ & $\begin{array}{l}-0.2330 \\
(0.0011) \\
\end{array}$ & $\begin{array}{c}0.0580 \\
(0.4240) \\
\end{array}$ & $\begin{array}{c}0.3935 \\
(0.0000) \\
\end{array}$ & $\begin{array}{c}0.2847 \\
(0.0001) \\
\end{array}$ & $\begin{array}{c}0.1189 \\
(0.1181) \\
\end{array}$ & $\begin{array}{c}0.1451 \\
(0.0561) \\
\end{array}$ & $\begin{array}{c}0.2450 \\
(0.0010) \\
\end{array}$ & & & & & & & \\
\hline Gcgksv & $\begin{array}{l}-0.3885 \\
(0.0000) \\
\end{array}$ & $\begin{array}{c}0.8011 \\
(0.0000) \\
\end{array}$ & $\begin{array}{c}0.2687 \\
(0.0002) \\
\end{array}$ & $\begin{array}{l}-0.2495 \\
(0.0005) \\
\end{array}$ & $\begin{array}{l}-0.0253 \\
(0.7278) \\
\end{array}$ & $\begin{array}{c}0.2265 \\
(0.0016) \\
\end{array}$ & $\begin{array}{c}0.1266 \\
(0.0803) \\
\end{array}$ & $\begin{array}{r}-0.0177 \\
(0.8169) \\
\end{array}$ & $\begin{array}{c}0.1774 \\
(0.0192) \\
\end{array}$ & $\begin{array}{l}-0.0535 \\
(0.4795 \\
\end{array}$ & $\begin{array}{c}0.6994 \\
(0.0000) \\
\end{array}$ & & & & & & \\
\hline Saves & $\begin{array}{l}-0.2376 \\
(0.0000) \\
\end{array}$ & $\begin{array}{c}0.0989 \\
(0.1724) \\
\end{array}$ & $\begin{array}{c}0.1346 \\
(0.0626) \\
\end{array}$ & $\begin{array}{c}0.1322 \\
(0.0676) \\
\end{array}$ & $\begin{array}{c}0.0138 \\
(0.8495) \\
\end{array}$ & $\begin{array}{l}-0.0917 \\
(0.2059) \\
\end{array}$ & $\begin{array}{l}-0.0213 \\
(0.7689) \\
\end{array}$ & $\begin{array}{c}0.0618 \\
(0.4177)\end{array}$ & $\begin{array}{c}0.1195 \\
(0.1162) \\
\end{array}$ & $\begin{array}{l}-0.2079 \\
(0.0055) \\
\end{array}$ & $\begin{array}{l}-0.5510 \\
(0.0000) \\
\end{array}$ & $\begin{array}{c}0.1248 \\
(0.0846) \\
\end{array}$ & & & & & \\
\hline Gkct & $\begin{array}{c}0.1719 \\
(0.0221) \\
\end{array}$ & $\begin{array}{l}-0.1265 \\
(0.0934) \\
\end{array}$ & $\begin{array}{c}0.2905 \\
(0.0001) \\
\end{array}$ & $\begin{array}{c}0.2517 \\
(0.0007) \\
\end{array}$ & $\begin{array}{c}0.1791 \\
(0.0170) \\
\end{array}$ & $\begin{array}{c}0.2251 \\
(0.0026) \\
\end{array}$ & $\begin{array}{c}0.3340 \\
(0.0000) \\
\end{array}$ & $\begin{array}{c}0.2654 \\
(0.0007) \\
\end{array}$ & $\begin{array}{l}-0.1258 \\
(0.1141) \\
\end{array}$ & $\begin{array}{c}0.4301 \\
(0.0000) \\
\end{array}$ & $\begin{array}{c}0.0684 \\
(0.3654) \\
\end{array}$ & $\begin{array}{c}-0.0308 \\
(0.6842) \\
\end{array}$ & $\begin{array}{c}-0.0159 \\
(0.8333) \\
\end{array}$ & & & & \\
\hline Tackles & $\begin{array}{c}0.0361 \\
(0.6195) \\
\end{array}$ & $\begin{array}{c}0.0422 \\
(0.5608) \\
\end{array}$ & $\begin{array}{c}0.3878 \\
(0.0000)\end{array}$ & $\begin{array}{c}0.0190 \\
(0.7937) \\
\end{array}$ & $\begin{array}{c}0.3138 \\
(0.0000)\end{array}$ & $\begin{array}{c}0.6497 \\
(0.0000) \\
\end{array}$ & $\begin{array}{c}0.3619 \\
(0.0000)\end{array}$ & $\begin{array}{c}0.6835 \\
(0.0000)\end{array}$ & $\begin{array}{c}0.5016 \\
(0.0000)\end{array}$ & $\begin{array}{c}0.7148 \\
(0.0000)\end{array}$ & $\begin{array}{c}0.2903 \\
(0.0000) \\
\end{array}$ & $\begin{array}{c}0.0702 \\
(0.3330) \\
\end{array}$ & $\begin{array}{c}-0.1530 \\
(0.0341) \\
\end{array}$ & $\begin{array}{c}0.2972 \\
(0.0001) \\
\end{array}$ & & & \\
\hline Yellow & $\begin{array}{l}-0.3390 \\
(0.0000) \\
\end{array}$ & $\begin{array}{c}0.1862 \\
(0.0097) \\
\end{array}$ & $\begin{array}{l}-0.0811 \\
(0.2634) \\
\end{array}$ & $\begin{array}{l}-0.0622 \\
(0.3916) \\
\end{array}$ & $\begin{array}{l}-0.1714 \\
(0.0175) \\
\end{array}$ & $\begin{array}{c}-0.1240 \\
(0.0865) \\
\end{array}$ & $\begin{array}{l}-0.2485 \\
(0.0005) \\
\end{array}$ & $\begin{array}{l}-0.3519 \\
(0.0000) \\
\end{array}$ & $\begin{array}{c}0.0169 \\
(0.8244) \\
\end{array}$ & $\begin{array}{l}-0.3557 \\
(0.0000) \\
\end{array}$ & $\begin{array}{c}0.0040 \\
(0.9557) \\
\end{array}$ & $\begin{array}{c}0.1638 \\
(0.0232) \\
\end{array}$ & $\begin{array}{c}0.0427 \\
(0.5562) \\
\end{array}$ & $\begin{array}{l}-0.2909 \\
(0.0001) \\
\end{array}$ & $\begin{array}{l}-0.2657 \\
(0.0002) \\
\end{array}$ & & \\
\hline Red & $\begin{array}{l}-0.3053 \\
(0.0000) \\
\end{array}$ & $\begin{array}{c}0.1254 \\
(0.0832) \\
\end{array}$ & $\begin{array}{c}0.0302 \\
(0.6772) \\
\end{array}$ & $\begin{array}{c}0.0491 \\
(0.4987) \\
\end{array}$ & $\begin{array}{l}-0.0674 \\
(0.3326) \\
\end{array}$ & $\begin{array}{c}0.1278 \\
(0.0772) \\
\end{array}$ & $\begin{array}{c}-0.0212 \\
(0.7707) \\
\end{array}$ & $\begin{array}{l}-0.1067 \\
(0.1613) \\
\end{array}$ & $\begin{array}{c}0.0831 \\
(0.2758) \\
\end{array}$ & $\begin{array}{l}-0.0950 \\
(0.2085) \\
\end{array}$ & $\begin{array}{l}-0.0220 \\
(0.7624) \\
\end{array}$ & $\begin{array}{c}0.0902 \\
(0.2135) \\
\end{array}$ & $\begin{array}{c}0.1119 \\
(0.1222) \\
\end{array}$ & $\begin{array}{l}-0.0139 \\
(0.8545) \\
\end{array}$ & $\begin{array}{l}-0.0705 \\
(0.3313) \\
\end{array}$ & $\begin{array}{c}0.3655 \\
(0.0000) \\
\end{array}$ & \\
\hline Fouls & $\begin{array}{l}-0.0578 \\
(0.4258) \\
\end{array}$ & $\begin{array}{c}0.0582 \\
(0.4225) \\
\end{array}$ & $\begin{array}{c}0.2745 \\
(0.0001) \\
\end{array}$ & $\begin{array}{c}0.0815 \\
(0.2614) \\
\end{array}$ & $\begin{array}{c}0.5711 \\
(0.0000) \\
\end{array}$ & $\begin{array}{c}0.4406 \\
(0.0000) \\
\end{array}$ & $\begin{array}{c}0.7061 \\
(0.0000) \\
\end{array}$ & $\begin{array}{c}0.6804 \\
(0.0000) \\
\end{array}$ & $\begin{array}{c}0.5011 \\
(0.0000) \\
\end{array}$ & $\begin{array}{c}0.5367 \\
(0.0000) \\
\end{array}$ & $\begin{array}{c}0.0811 \\
(0.2633) \\
\end{array}$ & $\begin{array}{c}0.0657 \\
(0.3653) \\
\end{array}$ & $\begin{array}{c}0.1309 \\
(0.0704) \\
\end{array}$ & $\begin{array}{l}0.3113 \\
(0.000) \\
\end{array}$ & $\begin{array}{c}0.5443 \\
(0.0000) \\
\end{array}$ & $\begin{array}{l}-0.0377 \\
(0.6033) \\
\end{array}$ & $\begin{array}{c}0.0928 \\
(0.2006) \\
\end{array}$ \\
\hline
\end{tabular}


In order to utilise the dataset more efficiently, the number of independent variables needs to be reduced by creating composite variables to reflect implicitly latent and unobserved aspects of overall playing performance, thereby decreasing the degrees of freedom and eventually reducing problems linked to multicollinearity that could lead to instability in the parameters' estimates. Traditionally, researchers have used their knowledge of the sport in question, which implies an element of subjective judgement in the weighting of the components. In our case, we have included objective technical indicators that offer the most accurate idea of the teams' quality, structure and game style. Firstly, given the availability of this rich set of direct and meaningful performance measures, all the selected inputs were correlated and statistically significant with the relevant output measures (i.e. goals scored and $1 /$ goals conceded) accordingly. Shooting is probably the most important attacking skillset, as the number of goals scored is directly related to the intensity (shots) and accuracy of shooting (shots on target). When we consider a team's capacity to attack, passing skills are essential for to create goal-scoring opportunities. The passing parameters (assists, passes completed, balls touched) measure not only players' technical capabilities to move the ball from one area of the pitch to another, but also the players' reading of the game. In general, more passes and better passing accuracy would lead to more chances created. However, in modern football, passing the ball around just for the sake of keeping possession is often less effective than a fast-paced counter attack. Other attacking parameters include the capability to complete crosses (completed, in total, and as a ratio of both), corners and dribbles, which are expected to have a positive effect on the number of chances created. Defensive parameters have a high influence on the opponent's capability to create chances or to score goals. The capability to execute tackles, clearances, and interceptions interrupt the build-up of the opponent. As the last line of defence, the quality of the goalkeeper is taken into consideration; the saving parameters are expected to have a significant negative effect on the number of goals conceded. Secondly, those inputs that were highly correlated with other similar inputs, like assists, crosses, recovered balls and fouls committed, were discarded through a correlation test as shown in Tables 2 and 3. Then, we also eliminated those potential inputs that are subject to randomness, hazard or luck. Finally, we process a backward selection running regressions using the equations in function of the remaining and respective offensive and defensive inputs. The selected inputs are generally (but not necessarily) statistically significant (at uni- and multivariable analyses) and positively correlated with the relevant offensive and defensive output measures. Following these criteria, the chosen inputs reasonably resemble the attacking or defending collective 
and/or individual quality of football teams. Five offensive inputs and five defensive inputs were chosen respectively: shots, counter attacks, crosses completed, passes completed, and useful dribbles; saves, anticipations, tackles, clearances and opponents' offside.

\section{Efficiency and effectiveness and their classification}

In this section, we present the results obtained with the DEA models on offensive and defensive efficiencies and football effectiveness based on the inputs and outputs selected in the previous section. Then, Serie A clubs are ranked in terms of their offensive and defensive efficiency and football effectiveness in the league over the ten seasons, respectively in Table 4, Table 5 and Table 6 below. This analysis explains teams' playing behaviour in relation to the league final position. If a team is very efficient offensively and defensively, then it usually obtains a good final league ranking as the table shows. Although the strong correlation between these rankings is pretty robust, as highlighted in Table 5, there are some interesting cases in the rankings. For example, Roma in season 2008/09 and Livorno in season 2004/05 appear to have secured a favourable league position, given their poor use of defensive resources. Conversely, Chievo in season 2001/02, despite being efficient offensively as well as defensively, did not manage to do better than $5^{\text {th }}$ in the final league ranking.

Table 4: Offensive efficiency ranks (2000-2010)

\begin{tabular}{|c|c|c|c|c|c|c|c|c|c|}
\hline Team & Season & Top 10 & $\begin{array}{l}\text { League } \\
\text { position }\end{array}$ & $\begin{array}{l}\text { League } \\
\text { points }\end{array}$ & Team & Season & $\begin{array}{c}\text { Bottom } \\
10\end{array}$ & $\begin{array}{l}\text { League } \\
\text { position }\end{array}$ & $\begin{array}{l}\text { League } \\
\text { points }\end{array}$ \\
\hline Inter & $2007 / 08$ & 100 & 1 & 91 & Treviso & $2005 / 06$ & 43.8 & 19 & 21 \\
\hline Roma & $2003 / 04$ & 100 & 2 & 71 & Torino & $2006 / 07$ & 48.1 & 16 & 40 \\
\hline Chievo & $2001 / 02$ & 100 & 5 & 54 & Torino & $2002 / 03$ & 48.4 & 18 & 21 \\
\hline Milan & $2008 / 09$ & 100 & 3 & 74 & Livorno & $2009 / 10$ & 49.6 & 20 & 29 \\
\hline Milan & $2005 / 06$ & 100 & 2 & 88 & Empoli & $2003 / 04$ & 49.8 & 17 & 30 \\
\hline Roma & $2000 / 01$ & 100 & 1 & 75 & Modena & $2003 / 04$ & 51.8 & 16 & 30 \\
\hline Juventus & $2007 / 08$ & 100 & 3 & 72 & Ancona & $2003 / 04$ & 51.9 & 18 & 13 \\
\hline Inter & $2006 / 07$ & 100 & 1 & 97 & Lecce & $2005 / 06$ & 52.8 & 18 & 29 \\
\hline Juventus & 2008/09 & 100 & 2 & 74 & Siena & 2008/09 & 53.3 & 14 & 44 \\
\hline Inter & $2002 / 03$ & 100 & 2 & 65 & Reggina & 2008/09 & 54.7 & 19 & 31 \\
\hline
\end{tabular}

Table 5: Defensive efficiency ranks (2000-2010)

\begin{tabular}{ccccccccc}
\hline Team & Season & Top 10 & $\begin{array}{c}\text { League } \\
\text { position }\end{array}$ & $\begin{array}{c}\text { League } \\
\text { points }\end{array}$ & Team & Season & $\begin{array}{c}\text { Bottom } \\
10\end{array}$ & $\begin{array}{c}\text { League } \\
\text { position }\end{array}$ \\
\hline Inter & $2007 / 08$ & 100 & 1 & 91 & Bari & $2000 / 01$ & 39.1 & 18 \\
points
\end{tabular}


Table 6: Effectiveness ranks (2000-2010)

\begin{tabular}{|c|c|c|c|c|c|c|c|c|c|}
\hline Team & Season & Top 10 & $\begin{array}{l}\text { League } \\
\text { position }\end{array}$ & $\begin{array}{c}\text { League } \\
\text { points }\end{array}$ & Team & Season & $\begin{array}{c}\text { Bottom } \\
10\end{array}$ & $\begin{array}{l}\text { League } \\
\text { position }\end{array}$ & $\begin{array}{c}\text { League } \\
\text { points }\end{array}$ \\
\hline Milan & $2005 / 06$ & 100 & 2 & 88 & Venezia & $2001 / 02$ & 15.7 & 18 & 18 \\
\hline Inter & $2006 / 07$ & 100 & 1 & 97 & Ancona & $2003 / 04$ & 17 & 18 & 13 \\
\hline Inter & $2004 / 05$ & 94.6 & 3 & 72 & Torino & $2002 / 03$ & 21.2 & 18 & 21 \\
\hline Roma & $2007 / 08$ & 93.9 & 2 & 82 & Treviso & $2005 / 06$ & 23.5 & 20 & 21 \\
\hline Juventus & $2005 / 06$ & 93.4 & 1 & 91 & Bari & $2000 / 01$ & 23.8 & 18 & 20 \\
\hline Inter & 2008/09 & 90.6 & 1 & 84 & Messina & $2006 / 07$ & 24.7 & 20 & 26 \\
\hline Juventus & $2004 / 05$ & 89.3 & 1 & 86 & Como & $2002 / 03$ & 25 & 17 & 24 \\
\hline Fiorentina & $2007 / 08$ & 87.1 & 4 & 66 & Lecce & $2005 / 06$ & 25.4 & 19 & 29 \\
\hline Juventus & $2003 / 04$ & 86.1 & 1 & 72 & Fiorentina & 2001/02 & 27.6 & 17 & 22 \\
\hline Milan & $2003 / 04$ & 85.7 & 3 & 61 & Modena & $2003 / 04$ & 29.2 & 16 & 30 \\
\hline
\end{tabular}

In Table 7 below, we provide the mean and standard deviation of offensive and defensive efficiencies for each season. Figure 2 displays the box plots over the 10 seasons.

Table 7: Average offensive, defensive efficiencies and effectiveness in the Italian Serie A (2000-2010)

\begin{tabular}{lcccccc}
\hline \multirow{2}{*}{$\begin{array}{c}\text { Offensive efficiency } \\
\text { Mean }\end{array}$} & Std. Dev. & Mean & Std. Dev. & Mean & Std. Dev. \\
\hline $2000 / 01$ & 0.816 & 0.128 & 0.636 & 0.175 & 0.524 & 0.152 \\
$2001 / 02$ & 0.802 & 0.138 & 0.656 & 0.176 & 0.520 & 0.162 \\
$2002 / 03$ & 0.782 & 0.159 & 0.617 & 0.159 & 0.540 & 0.168 \\
$2003 / 04$ & 0.803 & 0.176 & 0.614 & 0.174 & 0.529 & 0.193 \\
$2004 / 05$ & 0.753 & 0.113 & 0.623 & 0.177 & 0.555 & 0.169 \\
$2005 / 06$ & 0.789 & 0.157 & 0.618 & 0.163 & 0.589 & 0.218 \\
$2006 / 07$ & 0.743 & 0.133 & 0.631 & 0.115 & 0.546 & 0.204 \\
$2007 / 08$ & 0.785 & 0.128 & 0.610 & 0.135 & 0.574 & 0.198 \\
$2008 / 09$ & 0.785 & 0.136 & 0.615 & 0.135 & 0.601 & 0.172 \\
$2009 / 10$ & 0.807 & 0.141 & 0.605 & 0.117 & 0.570 & 0.162 \\
\hline
\end{tabular}

Some preliminary findings can be extrapolated. Firstly, on average, the indicators for defensive efficiency are lower than those for the offensive efficiency along the ten seasons, implying that the "average Serie A team" was closer to the frontier of offensive efficiency than to that of defensive efficiency. However, the standard deviation of defensive efficiency indicates greater differences between Italian teams than for offensive efficiency. These results are in line with by Boscá et al. (2009). Secondly, over the ten season period, both offensive and defensive efficiencies have fluctuating trends. In other words, we see a change in clubs' efficiencies in Italian football. Thirdly, season 2006/07 after the Calciopoli scandal registers the lowest levels of offensive efficiency, along with the standard deviation of offensive deficiency topping that of defensive efficiency. This might lead to the argument that clubs 
became less offensively efficient, taking into consideration the point deductions of some clubs and the relegation of FC Juventus, the most successful team in Serie A.

Figure 2: Offensive and defensive efficiencies' box plot in the Italian Serie A (2000-2010)

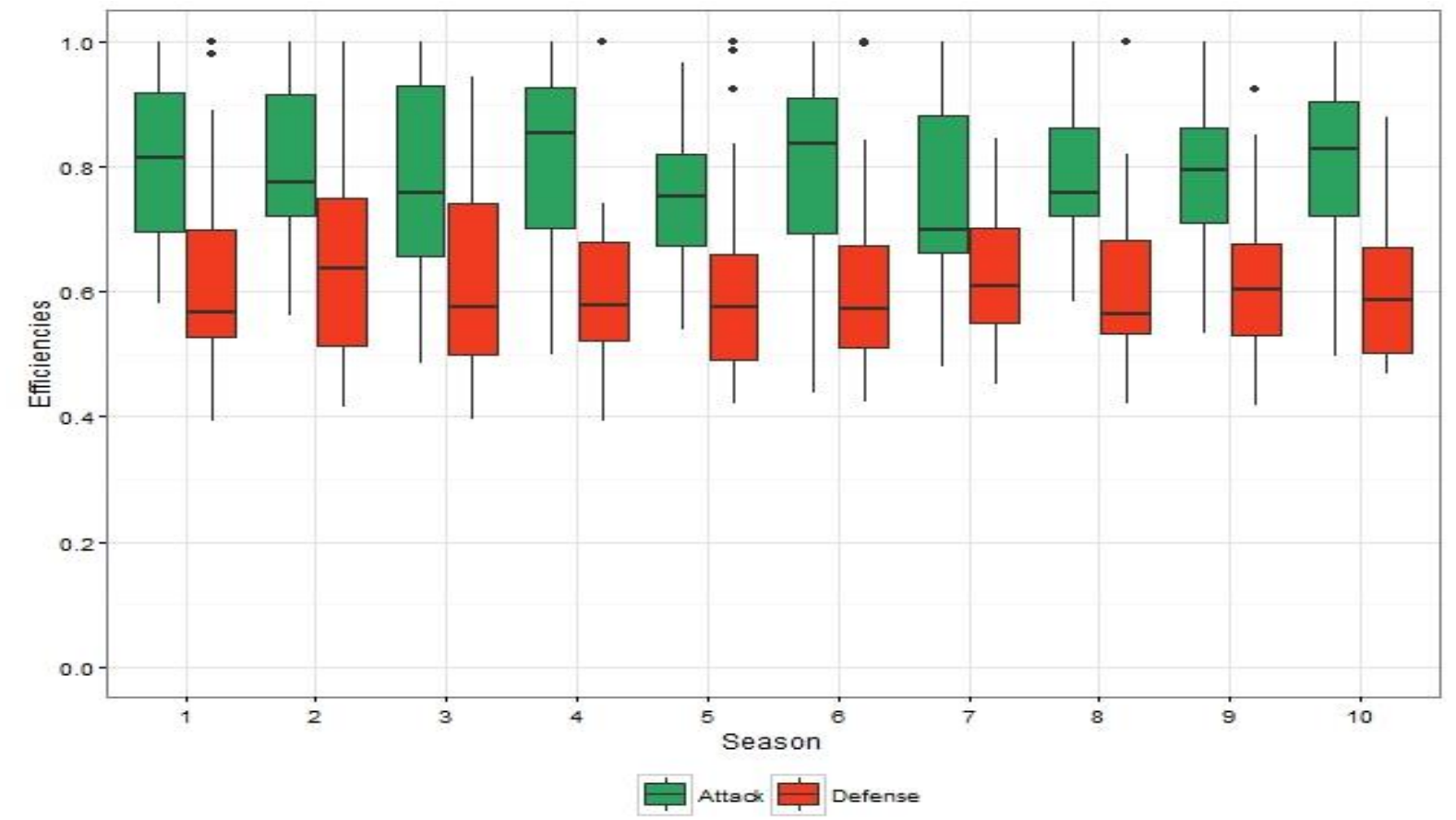

To support our DEA analysis about the relative average behaviour of teams in Italian football, Table 8 provides simple correlations between offensive and defensive efficiencies, between offensive efficiency and points, and between defensive efficiency and points. We use the number of points won as a percentage of the maximum winnable over the season, POINTS\%, as Serie A expanded from 18 to 20 clubs since the season 2004/05.

Table 8: Pearson correlation coefficients between points and indicators of offensive and defensive efficiencies in the Italian Serie A (2000-2010)

\begin{tabular}{lccc}
\hline Season & $\begin{array}{c}\text { Offensive efficiency and } \\
\text { defensive efficiency }\end{array}$ & $\begin{array}{c}\text { Offensive efficiency } \\
\text { and points }\end{array}$ & $\begin{array}{c}\text { Defensive efficiency } \\
\text { and points }\end{array}$ \\
\hline $2000 / 01$ & $\rho$ & $\rho$ & $\rho$ \\
$2001 / 02$ & 0.262 & $0.595^{* * *}$ & $0.753^{* * *}$ \\
$2002 / 03$ & $0.587^{*}$ & $0.592^{* * *}$ & $0.651^{* * *}$ \\
$2003 / 04$ & $0.557^{* *}$ & $0.777^{* * *}$ & $0.823^{* * *}$ \\
$2004 / 05$ & 0.346 & $0.673^{* * *}$ & $0.799^{* * *}$ \\
$2005 / 06$ & -0.119 & 0.349 & $0.620^{* * *}$ \\
$2006 / 07$ & $0.529^{* *}$ & $0.808^{* * *}$ & $0.802^{* * *}$ \\
$2007 / 08$ & $0.408^{* *}$ & $0.766^{* * *}$ & $0.773^{* * *}$ \\
$2008 / 09$ & $0.460^{* *}$ & $0.782^{* * *}$ & $0.747^{* * *}$ \\
$2009 / 10$ & $0.500^{* *}$ & $0.825^{* * *}$ & $0.676^{* * *}$ \\
\hline
\end{tabular}


Another interesting finding is that the correlations between offensive and defensive efficiency have been generally positive in all but one season (2004/2005): in five seasons the correlation coefficient was statistically significant at $\mathrm{p}<0.05$. In general, those Serie A teams that are relatively efficient offensively are also efficient defensively and vice-versa. This is also confirmed by the correlation between indicators of efficiency and points won in all the season (0.69 for offensive efficiency, 0.72 for defensive efficiency, both statistically significant at $\mathrm{p}<0.001)$. However, we can notice that, while at the beginning of the last decade, the largest correlations were scored between points won and the indicator of defensive efficiency, an opposite scenario is found at the second half of the decade, when the correlation between points won and the indicators of offensive efficiency have been generally higher. This finding suggests that there has been a change of tactical paradigm in Serie A, where a good attack has become a necessary condition to obtain the largest number of points, indicating that a team has to be more offensively, rather than defensively, efficient to win the Italian championship or avoid relegation to Serie B.

We use regression analysis to further explain the points obtained by teams during a season with different efficiency indicators and we can attempt to understand with greater precision how Italian football changed in the last decade.

Table 9: Points and efficiency indicators in the Italian Serie A season by season (OLS estimates)

\begin{tabular}{|c|c|c|c|c|c|c|c|c|c|c|}
\hline \multicolumn{11}{|c|}{ Dependent variable: Points\% } \\
\hline & $2000 / 01$ & $2001 / 02$ & $2002 / 03$ & 2003/04 & $2004 / 05$ & $2005 / 06$ & $2006 / 07$ & $2007 / 08$ & 2008/09 & $2009 / 10$ \\
\hline Constant & $-0.752^{*}$ & -0.213 & $-0.512^{* *}$ & $-0.843^{* * *}$ & -0.650 & $-1.64^{* *}$ & $-1.378^{* * *}$ & $-0.960^{* * *}$ & $-0.809^{* *}$ & $-1.050^{* * *}$ \\
\hline $\begin{array}{l}\text { Offensive } \\
\text { efficiency }\end{array}$ & $1.391^{* *}$ & 1.010 & $1.212^{* * *}$ & $1.297^{* *}$ & $1.470^{* *}$ & $1.676^{* * *}$ & $1.820^{* * *}$ & $1.784^{* * *}$ & $1.946^{* * *}$ & $1.647^{* * *}$ \\
\hline $\begin{array}{l}\text { Defensive } \\
\text { efficiency }\end{array}$ & $1.528^{* * *}$ & $1.158^{*}$ & $1.481^{* *}$ & $1.878^{* * *}$ & $1.407^{* * *}$ & $1.685^{* * *}$ & $2.176^{* * *}$ & $1.493^{* *}$ & $1.063^{*}$ & $1.792^{* * *}$ \\
\hline $\mathrm{R}^{2}$ & 0.737 & 0.489 & 0.825 & 0.817 & 0.597 & 0.867 & 0.842 & 0.803 & 0.774 & 0.741 \\
\hline $\begin{array}{l}\text { Number of } \\
\text { observation }\end{array}$ & 18 & 18 & 18 & 18 & 20 & 20 & 20 & 20 & 20 & 20 \\
\hline
\end{tabular}

Table 9 presents the results of linear regressions for the ten Serie A seasons that explain the points obtained by teams during a season in relation to the different efficiencies. There are several points that should be highlighted, indicating that the general tendencies for the Italian league have changed. An increase in defensive efficiency with 10 per cent will imply a gain 
ranging between 0.106 and 0.217 points per game, depending on the concrete championship we look at. These figures range between 0.101 and 0.194 if we look at the coefficients estimated for the general offensive efficiency variable. Thus, for an Italian team, the popular maxim supported by Boscá et al. (2009), the best attack begins with a good defence, does not hold. The regressions' results confirm that to explain points won along the ten seasons, the difference between coefficients associated with general defensive efficiency and general offensive efficiency moves from positive towards negative. In particular, this is notable since the season 2004/05 when Serie A expanded from 18 to 20 clubs and the coefficient associated with general offensive efficiency of 1.47 (95\% CI $[0.41 ; 2.53])$ is roughly similar to that of the defensive efficiency of 1.41 (95\% CI [0.73; 2.08]). Since then, the impact of offensive efficiency has become predominantly higher than the impact of defensive efficiency. However, if we look at these results in more detail, we can see that there remain some interpretative problems. For example, in the regression to explain points won in the 2006/07 season, a general coefficient of defensive efficiency of 2.17 is obtained, resulting in the highest level along the ten years. This peculiar result can be linked to the fact that the season 2006/07 was when the Calciopoli scandal decisions were inflicted as we test in the next part.

\section{Evaluation of the impact of the Calciopoli scandal on efficiency and effectiveness}

In table 10, estimations 1 and 2 are the specifications that formally test a structural break to assess the impact of the Calciopoli scandal. Our hypothesis is that Serie A clubs performance deteriorated following the Calciopoli sanctions. Two specific variables, Cal and Post-Cal, were inserted in our model to assess the trend before the Calcipoli Scandal and after the same scandal accordingly. In both estimations, the results are very similar and the larger absolute size of the coefficient on the attacking measure relative to the measure of defensive performance suggests that attacking play is the more important determinant of league success overall.

Both variables, $\mathrm{Cal}$ and Post-Cal, are statistically significant but with a divergent sign. This evidence suggests that, during the season of Calciopoli (2006-07), we reach a positive peak in the relationship between Points\% against offensive and defensive efficiencies that is counter-balanced by a decline of the same relationship during the following seasons after Calciopoli. In other words, we witness a structural break in Italian football during 
the Calciopoli scandal that had an impact on clubs' offensive and defensive efficiencies and effectiveness.

Table 10: Multilevel linear regressions between points and indicators of offensive and defensive efficiencies in the Italian Serie A (2000-2010)

\begin{tabular}{|c|c|c|}
\hline & \multicolumn{2}{|c|}{$\begin{array}{c}\text { Dependent variable } \\
2000 / 01-2009 / 10\end{array}$} \\
\hline & Points\% (1) & Points\% (2) \\
\hline Offensive efficiency & $1.185^{* * *}$ & $1.175^{* * *}$ \\
\hline Defensive efficiency & $1.146^{* * *}$ & $1.152^{* * *}$ \\
\hline Season & & $0.015^{*}$ \\
\hline Cal & & $0.237^{*}$ \\
\hline Post-Cal & & $-0.338^{*}$ \\
\hline $2001 / 02$ & 0.039 & \\
\hline $2002 / 03$ & 0.084 & \\
\hline $2003 / 04$ & 0.052 & \\
\hline $2004 / 05$ & 0.088 & \\
\hline $2005 / 06$ & 0.085 & \\
\hline $2006 / 07$ & $0.130^{*}$ & \\
\hline $2007 / 08$ & 0.062 & \\
\hline 2008/09 & 0.090 & \\
\hline $2009 / 10$ & 0.058 & \\
\hline LR test & $57.10^{* * *}$ & $55.99^{* * *}$ \\
\hline Number of observations & & 192 \\
\hline
\end{tabular}

To better analyse the behaviour of the clubs involved in the Calciopoli scandal, Table 11 and Table 12 compare respectively the offensive and defensive efficiency rankings of the teams that competed in season 2005/06 or season 2006/07. Apart from Reggina Calcio, all the other teams involved in the Calciopoli scandal scored higher with respect to offensive efficiency in season 2005/06 than in season 2006/07 as shown in Table 11. While in the 2005/06 season ACF Fiorentina and AC Milan were the most efficient teams offensively and Lazio was ranked $6^{\text {th }}$, the same teams had a worse offensive efficiency performance the following year when the points deductions were inflicted. Only Reggina Calcio registered an increase of offensive efficiency that was the highest achieved along the ten seasons. Being always involved in the battle to avoid relegation, the club might have understood that the best strategy was to adopt a more offensive playing style to recover the points deduction.

In Table 12, we see an opposite scenario. Except for AC Milan, all the clubs involved in the scandal improved their defensive efficiency in the season after the Calciopoli scandal. 
In particular, these clubs also registered their highest level of defensive efficiency along the ten seasons period.

Table 11: Offensive efficiency ranks (2005-2007)

\begin{tabular}{lclc}
\hline Club & $\begin{array}{c}\text { Offensive efficiency } \\
2005 / 06\end{array}$ & Club & $\begin{array}{c}\text { Offensive efficiency } \\
2006 / 07\end{array}$ \\
\hline Juventus & 0.916 & Inter & 1.000 \\
Milan & 1.000 & Roma & 0.936 \\
Inter & 0.851 & Lazio & 0.892 \\
Fiorentina & 1.000 & Milan & 0.692 \\
Roma & 0.942 & Palermo & 0.821 \\
Lazio & 0.909 & Fiorentina & 0.891 \\
Chievo & 0.954 & Empoli & 0.692 \\
Palermo & 0.734 & Atalanta & 0.890 \\
Livorno & 0.832 & Sampdoria & 0.742 \\
Parma & 0.844 & Udinese & 0.704 \\
Empoli & 0.842 & Livorno & 0.685 \\
Ascoli & 0.745 & Parma & 0.647 \\
Udinese & 0.682 & Catania & 0.736 \\
Sampdoria & 0.644 & Reggina Calcio & 0.880 \\
Reggina Calcio & 0.865 & Siena & 0.613 \\
Cagliari & 0.790 & Torino & 0.408 \\
Siena & 0.698 & Cagliari & 0.595 \\
Messina & 0.572 & Chievo & 0.667 \\
Lecce & 0.528 & Ascoli & 0.688 \\
Treviso & 0.438 & Messian & 0.604 \\
\hline \multicolumn{2}{c}{ Note: The teams are ranked according to their final league ranking. } \\
& In bold teams involved in Calciopoli scandal. & \\
& & &
\end{tabular}

Table 12: Defensive efficiency ranks (2005-2007)

\begin{tabular}{lclc}
\hline Club & $\begin{array}{c}\text { Defensive efficiency } \\
2005 / 06\end{array}$ & Club & $\begin{array}{c}\text { Defensive efficiency } \\
2006 / 07\end{array}$ \\
\hline Juventus & 1.000 & Inter & 0.795 \\
Milan & 0.843 & Roma & 0.788 \\
Inter & 0.998 & Lazio & 0.796 \\
Fiorentina & 0.717 & Milan & 0.474 \\
Roma & 0.589 & Palermo & 0.679 \\
Lazio & 0.660 & Fiorentina & 0.844 \\
Chievo & 0.603 & Empoli & 0.638 \\
Palermo & 0.493 & Atalanta & 0.483 \\
Livorno & 0.732 & Sampdoria & 0.548 \\
Parma & 0.523 & Udinese & 0.575 \\
Empoli & 0.512 & Livorno & 0.552 \\
Ascoli & 0.573 & Parma & 0.543 \\
Udinese & 0.515 & Catania & 0.451 \\
Sampdoria & 0.423 & Reggina Calcio & 0.578 \\
Reggina Calcio & 0.535 & Siena & 0.624 \\
Cagliari & 0.505 & Torino & 0.685 \\
Siena & 0.579 & Cagliari & 0.671 \\
Messina & 0.478 & Chievo & 0.592 \\
Lecce & 0.573 & Ascoli & 0.562 \\
Treviso & 0.505 & Messian & 0.479 \\
\hline
\end{tabular}

Note: The teams are ranked according to their final league ranking.

In bold, teams involved in Calciopoli scandal.

A plausible explanation to the case of AC Milan is that the club received the highest points deduction and it was also competing in the UEFA Champions League. The club was aware that its chance for the Serie A title was almost null and that the only ambition for that season was to qualify for the next UEFA Champions League. Thus, out of the five Serie A teams implicated in Calciopoli, ACF Fiorentina, Reggina Calcio and SS 
Lazio appeared to have accrued points more efficiently in defence in 2006/07 than they did along the other seasons and this might be indicative of the impact of the points deductions. These results corroborate the main finding of our analysis, that the Italian top professional football league has displayed a change of playing style.

Table 13: Effectiveness ranks (2005-2007)

\begin{tabular}{lclc}
\hline Club & $\begin{array}{c}\text { Effectiveness } \\
2005 / 06\end{array}$ & Club & $\begin{array}{c}\text { Effectiveness } \\
2006 / 07\end{array}$ \\
\hline Juventus & 93.4 & Inter & 100 \\
Milan & 100 & Roma & 85.7 \\
Inter & 84.9 & Lazio & 66.5 \\
Fiorentina & 81.7 & Milan & 85.2 \\
Roma & 80.4 & Palermo & 59.3 \\
Lazio & 71.0 & Fiorentina & 74.7 \\
Chievo & 63.0 & Empoli & 65.3 \\
Palermo & 52.4 & Atalanta & 57.4 \\
Livorno & 42.7 & Sampdoria & 56.2 \\
Parma & 61.0 & Udinese & 57.5 \\
Empoli & 50.9 & Livorno & 49.1 \\
Ascoli & 50.1 & Parma & 44.0 \\
Udinese & 37.5 & Catania & 46.3 \\
Sampdoria & 73.0 & Reggina Calcio & 59.3 \\
Reggina Calcio & 50.4 & Siena & 37.7 \\
Cagliari & 50.3 & Torino & 34.9 \\
Siena & 54.7 & Cagliari & 35.6 \\
Messina & 31.5 & Chievo & 34.0 \\
Lecce & 25.4 & Ascoli & 29.9 \\
Treviso & 23.5 & Messian & 24.7 \\
\hline Note: The teams are ranked according to their final league ranking. & \\
& In bold, teams involved in Calciopoli scandal. &
\end{tabular}

\section{Implications and conclusions}

This paper has focused on the on-field performance of Serie A football clubs over the last decade in order to analyse their production function in terms of efficiency and effectiveness. The analysis included those seasons scarred by the Calciopoli scandal to assess its impact on clubs' performance. Based on a two-stage DEA approach, attacking and defensive playing efficiencies were modelled as inputs in the production of league level success.

Our results partially confirmed results by by Boscá et al. (2009) that in Italy an efficient defence was the best way to obtain the most points. However, according to our estimations, increasing offensive efficiency pays relatively more than increasing defensive efficiency since the 2005/06 season. If we look at these results in detail, the punishments imposed on the implicated clubs in Calciopoli do appear to have affected actual defensive and offensive performance. At least three of these clubs - Reggina, Lazio and Fiorentina - appear to have performed worse defensively in season 2005/06, where they were judged to have cheated, than in the subsequent season, where they were punished. Conversely, Reggina was the only 
club to increase its offensive efficiency, while AC Milan underperformed both defensively and offensively compared to the previous season.

This study has managerial implications for the clubs, as well as for the league authorities. Our results indicate that it in order to be more effective, it is more important for Serie A clubs to be offensively than defensively efficient. Indeed, contrary to common belief, it is nowadays more rewarding for Serie A teams to invest in high-quality attacking players rather than tough defenders. Club managers can use this knowledge in their strategy on the transfer market or in negotiations on player salaries. League authorities should be aware that sanctions regarding future seasons, in addition to being a way to compensate for undue advantages and to deter other clubs from committing fraud, can also have an impact on the playing style and performance. Indeed, our analysis suggests that the sanctioned clubs took short-term decisions away from their usual tactical behaviour and presumably adopted different playing strategies to compensate for the impact of the points deductions. In turn, this may have an impact on the attractiveness of the league as a whole, which is a key aspect for public interest, broadcasting revenues and sponsorship deals.

After the Calciopoli scandal, many Italian clubs, which were used to spending so much money on good offensive and defensive players, have also faced the loss of competitive advantage over other European leagues. This may also have affected the transfer market strategies of Italian clubs and consequently their playing style. The above results call for a rigorous cost-benefit analysis of this change and the temptation of adopting corruptive behaviour in professional football. Top clubs such as Juventus and AC Milan have budgets that are usually several times larger than any medium or small club and their player transfer market continues to expand these differences in the relative values of defending and attacking players. If we include these financial gaps between clubs, relative differences could be greater than those measurable by any indicator of offensive and defensive efficiency. This aspect can be related to how costly the adoption of fraudulent behaviour can be to individual clubs and the league as a whole. Indeed, the costs associated with relegation, or the loss of competing in European cups, may well have a relevant impact on a clubs' financial stability, providing incentives to adopt fraudulent behaviour.

To conclude, further research should expand its horizon by including more European leagues. A comparative approach would provide interesting findings and better explain how 
clubs' strategies and tactics vary league by league, as we always assume that football in each country is inspired and affected by different social, cultural and economic factors.

\section{References}

Agnew, P. (2007). Forza Italia [Go Italy]. London: Random House.

Arel, PricewaterhouseCoopers, \& Federcalcio (2012). Report Calcio. Rome: AREL.

Asmild, M., Paradi, J. C., Reese, D. N. and Tam, F. (2007). Measuring overall Efficiency and Effectiveness using DEA. European Journal of Operational Research, 178, pp. 305-321.

Atkinson, S. E., Stanley, L. R., \& Tschirart, J. (1988). Revenue Sharing as an Incentive in an Agency Problem: An Example from the National Football League. The RAND Journal of Economics, 19, 27-43.

Babatunde, B., Migali, S., \& Simmons, R. (2012) Corruption does not pay: An Analysis of Consumer Response to Italy's Calciopoli Scandal. Oxford Bulletin of Economics and Statistics, $78,22-41$.

Baroncelli, A., \& Lago, U. (2006). Italian Football. Journal of Sports Economics, 7, 13-28.

Barros, C. P., Assaf, A., \& Sá-Earp, F. (2010). Brazilian Football League Technical Efficiency: A Simar and Wilson Approach. Journal of Sports Economics, 11, 641-651.

Barros, C. P., Del Corral, J., \& Garcia-del-Barrio, P. (2008b). Identification of Segments of Soccer Clubs in the Spanish League First Division with a Latent Class Model. Journal of Sports Economics, 9, 451-469

Barros, C. P., \& Garcia-del-Barrio, P. (2008). Efficiency Measurement of the English Football Premier League with a Random Frontier Model. Economic Modelling, 25, 994-1002.

Barros, C. P., Garcia-del-Barrio, P., \& Leach, S. (2009). Analysing the Technical Efficiency of the Spanish Football League First Division with a Random Frontier Model. Applied Economics, 41, 3239-3247.

Barros, C. P., \& Leach, S. (2006a). Performance Evaluation of the English Premier Football League with Data Envelopment Analysis. Applied Economics, 38, 1449-1458. 
Barros, C. P., \& Leach, S. (2006b). Analyzing the Performance of the F.A. English Premier League with an Econometric Frontier Model. Journal of Sports Economics, 7, 391-407.

Barros, C. P., \& Leach, S. (2007). Technical Efficiency in the English Football Association Premier League with a Stochastic Cost Frontier. Applied Economics Letters, 14, 731-741.

Boeri, T., \& Severgini, B. (2011). Match Rigging and the Career Concerns of Referees. Labour Economics, 18, 349-359.

Boeri, T., \& Severgini, B. (2012). The Decline of Professional Football in Italy. Discussion Paper Series, Forschungsinstitut zur Zukunt der Arbeit, No. 7018.

Bof, F., Montanari, F., \& Silvestri, G. (2008). Il Management del Calcio [Football Management]. Milano: Franco Angeli Editore.

Boscá, J. E., Liern, V., Martinez, A., \& Sala, R. (2009). Increasing Offensive and Defensive Efficiency? An Analysis of Italian and Spanish Football. Omega: The International Journal of Management Science, 37, 63-78.

Brittan, D. (1997). Business Lessons from Darwin. MIT Reporter, 12(1), pp. 12-23.

Carmichael, F., McHale, I., \& Thomas, D. (2010). Maintaining market position: team performance, revenue and wage expenditure in the English Premier League. Bulletin of Economic Research, 63(4), 464-497.

Carmichael, F., Rossi, G., \& Thomas, D. (2017). Production, Efficiency and Corruption in Italian Serie A Football. Journal of Sport Economics, 18, 34-57.

Carmichael, F., \& Thomas, D. (1995). Production and Efficiency in Team Sports: An Investigation of Rugby League Football. Applied Economics, 27, 859-869.

Carmichael, F., Thomas, D., \& Ward, R. (2000). Team Performance: The Case of English Premiership Football. Managerial and Decision Economics, 21, 31-45.

Carmichael, F., Thomas, D., \& Ward, R. (2001). Production and Efficiency in Association Football. Journal of Sports Economics, 2, 228-243.Caruso, R., \& Di Domizio, M. (2012). Hooliganism and Football Demand in Italy: Evidence for the Period 1962-2011,DISCE Quaderni dell'Istituto di Politica Economica, Ispe 062, Universitá Cattolica del Sacro Cuore, Dipartimenti ed Istituti di Scienze Economiche (DISCE). 
Chan, F. T. S. (2003). Performance Measurement in Supply Chain. The International Journal of Advanced Manufacturing Technology, 21, pp. 1351-1360.

Chatterjee, S., Campbell, M. R., \& Wiseman, F. (1994). Take that Jam! An Analysis of Winning Percentage for NBA Teams. Managerial and Decisions Economics, 15, 521-555.

Collier, T., Johnson, A. L., \& Ruggiero, J. (2011). Measuring Technical Efficiency in Sports. Journal of Sports Economics, 12, 579-598.

Dawson, P., \& Dobson, D. (2002). Managerial Efficiency and Human Capital: An Application to English Association Football. Managerial and Decision Economics, 23, 471-486.

Dawson, P., Dobson, S., \& Gerrard, B. (2000). Estimating Coaching Efficiency in Professional Team Sports: Evidence from English Association Football. Scottish Journal of Political Economy, 47, 399-421.

Di Meo, S., \& Ferraris, G., (2012). Il Pallone Criminale [Criminal Football]. Milano: Ponte alle Grazie.

Drucker, P. F. (1963). Managing for Business Effectiveness. Harward Business Review, 41, pp. 53-60.

Espitia-Escuer, M., \& García-Cebríán, L. (2004). Measuring the Efficiency of Spanish First Division Soccer Teams. Journal of Sports Economics, 5, 329-346.

Espitia-Escuer, M., \& García-Cebríán, L. I. (2006). Performance in Sports Teams: Results and Potential in the Professional Soccer League in Spain. Management Decision, 44, 1020-1030.

Espitia-Escuer, M., \& García-Cebríán, L. I. (2008). Measuring the Productivity of Spanish First Division Soccer Teams. European Sport Management Quarterly, 8, 229-246.

Foot, J., (2007). Calcio: A History of Italian Football. London: Harper Perennial.

Frick, B., \& Simmons, R. (2008). The Impact of Managerial Quality on Organizational Performance: Evidence from German Soccer. Managerial and Decision Economics, 29, 593600.

García-Sanchez, I. M. (2007). Efficiency and Effectiveness of Spanish Football Teams: A Three-stage DEA Approach. Central European Journal of Operations Research, 15, 21-45. 
Gerrard, B. (2006). Analysing the Win-Wage Relationship in Pro Sports Leagues: Evidence from the FA Premier League 1997/98 - 2001/02?, in: P. Rodriguez, S. Kesenne and J.Garcia (Eds.), Sports Economics After Fifty Years: Essays in Honour of Simon Rottenberg, Ediciones de la Universidad de Oviedo, Oviedo.

González-Gómez, F., \& Picazo-Tadeo, A. J. (2010). Can we be satisfied with our Football Team? Evidence from Spanish Professional Football. Journal of Sports Economics, 11, 418442.

Guzmán, I. \& Morrow, S. (2007). Measuring Efficiency and Productivity in Professional Football Teams: Evidence from the English Premier League. Central European Journal of Operations Research, 15, 309-328.

Haas, D. J. (2003a). Productive Efficiency of English Football Clubs: A Data Envelopment Analysis Approach. Managerial and Decision Economics, 24, 403-410.

Haas, D. J. (2003b). Technical Efficiency in the Major League Soccer. Journal of Sports Economics, 4, 203-215.

Haas, D. J., Kocher, M. G., \& Sutter, M. (2004). Measuring Efficiency of German Football Team by Data Envelopment Analysis. Central European Journal of Operations Research, 12, 251-268.

Hamil, S., Morrow, S., Idle, C., Rossi, G., \& Faccendini, S. (2010). The Governance and Regulation of Italian Football. Soccer and Society, 11, 373-413.

Jones, T. (2007). The Dark Heart of Italy. London: Faber \& Faber.

Krautmann, A. C. (1990). Shirking or Stochastic Productivity in Major League Baseball. Southern Economic Journal, 56, 567-579.

Ken, H. T., Chu, S. and Xu, J. (2006). Efficiency, Effectiveness and Productivity of Marketing in Services. European Journal of Operational Research, 170, pp. 265-276.

Korten, D. (1998). The Post-Corporate World - Life After Capitalism. San Francisco: BerretKohler.

Magretta, J. (2000). Managing in the New Economy. New York: Business Review Book. 
McCormick, R. E., \& Clement, R. C. (1992). Intra-firm Profit Opportunities and Managerial Slack: Evidence from Professional Basketball, in: Scully, G. W. (Ed.), Advances in the Economics of Sports, JAI Press, Greenwich.

Modis, T. (1998). Conquering Uncertainty: Understanding Corporate Cycle and Positioning Your Company to Survive the Changing Environment. New York: McGrow-Hill.

Porter, M. (1985). Competitive Advantage. New York: Free Press.

Porter, P. K., \& Scully, G. W. (1982). Measuring Managerial Efficiency: The Case of Baseball. Southern Economic Journal, 48, 642-650.

Rottenberg, S. (1956). The Baseball Players' Labour Market. Journal of Political Economy, $64,242-258$.

Ruggiero, J., Hadley, L., \& Gustafson, E. (1996). Technical Efficiency in Major League Baseball, in: Fizel, J., Gustafson, E. and Hadley, L. (Eds.), Sports Economics: Current Research, Praeger, Westport.

Sala-Garrido, R., Carrión, V. L., Esteve, A. M., \& Boscá, J .E. (2009). Analysis and Evolution of Efficiency in the Spanish Soccer League (2000/01-2007/08). Journal of Quantitative Analysis in Sports, 5, Article 3.

Schofield, J. A. (1988). Production Functions in the Sports Industry: An Empirical Analysis of Professional Cricket. Applied Economics, 15, 283-296.

Scott, F. A. J., Long, J. E., \& Somppi, K. (1985). Salary vs. Marginal Revenue Product under Monopsony and Competition: The Case of Professional Basketball. Atlantic Economic Journal, $13,50-59$.

Scott, R. (2000). Institutions and Organizations. New York: SAGE.

Scully, G. W. (1974). Pay and performance in Major League Baseball. American Economic Review, 64, 915-930.

Scully, G. W. (1989). The Business of Major League Baseball. Chicago: University of Chicago Press.

Scully, G. W. (1995). The Market Structure of Sports. Chicago: University of Chicago Press. 
Tiedemann, T., Francksen, T., \& Latacz-Lohmann, U. (2011). Assessing the Performance of German Bundesliga Football Players: A Non-parametric Metafrontier Approach. Central European Journal of Operations Research, 19, 571-587.

Villa, G. \& Lozano, S. (2016). Assessing the Scoring Efficiency of a Football Match. European Journal of Operational Research, 222, 559-569.

Zak, T. A., Huang, C. J., \& Sigfried, J. J. (1979). Production Efficiency: The Case of Professional Basketball. Journal of Business, 53, 379-302.

Zech, C. E. (1981). An Empirical Estimation of a Production Function: The Case of Major League Baseball. American Economist, 25, 19-23. 